correctional officers at the Toronto South Detention Centre (TSDC) and Vanier Centre for Women (VCW) in Ontario, Canada.

Method. A needs assessment was undertaken among officers at the TSDC. In response to needs identified, a one-day course was delivered to officers $(n=57)$ at the TSDC and VCW $(n=41)$. The curriculum included mental health awareness; assessment of risk; communicating with inmates in distress; and self-care. Live simulations provided the opportunity for participants to identify signs of mental illness, assess risk, and respond strategically to de-escalate situations. Participants' knowledge and confidence in their ability to identify and assist individuals with these problems was established using pre and post measures. Participant satisfaction was also measured via a survey. A threemonth follow-up administration was used to determine maintenance of gains. Focus groups at nine months were conducted to understand participants' needs, learning, and impact of training. Result. The results were promising, with $92 \%$ and $88 \%$ of participants at TSDC and Vanier Centre for Women respectively expressing satisfaction and $62 \%$ and $68 \%$ at TSDC and Vanier Centre for Women respectively stating they intended to change practices. Analyses of change in knowledge and confidence scores pre to post-training showed statistically significant improvement in all areas measured. Three-month follow-up at TSDC showed 75\% of respondents have applied what they learned from the training to a "moderate or great extent". Focus group themes showed improved attitudes and ability to identify behaviours related to inmate mental health struggles and interest in further training to support officers' mental health.

Conclusion. This study shows that training informed by officer learning needs can help them better meet the mental health needs of inmates. Training can improve attitudes toward inmates presenting with mental health issues. Training that is interactive and provides skills practice can have sustained impact on practice. Further training should integrate self-care to support officers' mental health.

\section{Exploring student thoughts and perception of videos as a learning resource}

\author{
Gianluca Di Pasqua* and Pranav Mahajan \\ University of Sheffield \\ ${ }^{\star}$ Corresponding author.
}

\section{doi: 10.1192/bjo.2021.381}

Aims. As medical education becomes increasingly digital, there is a plethora of readily available video resources available to medical students, aimed at teaching a wide range of topics. Despite this abundance, students report a myriad of issues. These range from videos containing outdated material, being of a poor production quality, and not being entirely relevant to their learning objectives. The aim of this study is to explore student thoughts and perceptions of videos as a teaching and learning resource. As the Mental State Examination is a component of the Psychiatry curriculum that students often find difficult, we have written, filmed and produced a video series explaining and demonstrating it.

Method. Following the production of the Mental State Examination videos at the University of Sheffield - which contained multiple doctor-patient consultations, interspersed with narration outlining the key learning points - three focus groups were undertaken. These were aimed at understanding student thoughts and perception on the new videos, and the use of videos in medical education in general. Taking a qualitative approach, thematic analysis was performed on the content of the focus groups.

Result. There was universal positive feedback about the structure and content of the videos we had produced; students enjoyed observing the various doctor-patient consultations and felt the separate elements of the Mental State Examination was explained logically and concisely. Furthermore, students appreciated that the videos were produced at their own University, believing this added to their validity. With regards to videos as a teaching resource in general, focus groups revealed that students appreciated specific, relevant and novel video material. Most students felt that videos can play an important role in medical education. There was a unanimous belief however that videos used in medical education should be produced well and be factually correct.

Conclusion. Understanding how students feel about videos as a teaching and learning resource is crucial in the development of more in the future. This study suggests more video resources for many areas, both within Psychiatry, and within the greater sphere of medical education, would be welcomed. This ought to be accompanied with student evaluation.

\section{The Safety Conversation: developing a trustwide safety conference at CNWL during a pandemic}

Emily Duncan*, Simon Edwards, Alison Butler and Cornelius Kelly

Central and Northwest London NHS Trust

${ }^{\star}$ Corresponding author.

doi: 10.1192/bjo.2021.382

Aims. The COVID pandemic has had both a massive impact on clinical service delivery and the way that training and education is provided. CNWL is a large NHS provider and has approximately 7000 staff working across 150 locations, providing mental health and community health services. In response to the need to share learning across the organisation, a trustwide "Safety Conversation Day" took place to spotlight the work being done to promote safety and to act as a platform to share ideas and learning across the trust. This was the first ever virtual conference organised by the trust.

Method. The one-day conference included virtual posters and an all-day open access virtual conversation delivered via zoom. The day was divided into 6 safety themes: Safety tools; Safer Environments; Supporting and Involving Staff; Safer use of Medicines; See Think Act and Relational Security; and Prevention is Better than Cure. Frontline staff delivered 5-6 short presentations each hour highlighting new ways of working, quality improvement, local research etc.

Staff were also encouraged to submit posters for the event, with webinars held on how to write a poster held prior to the safety conversation to promote engagement. Prizes were awarded for best posters in the different categories.

A mentimeter survey was running throughout the day to get feedback from participants.

Result. This was the largest event of this kind held by the trust. 430 unique viewers logged in during the day to watch the presentations.

Feedback was very positive on the mentimeter survey. 3 questions were asked on a likert scale of: Strongly Disagree - Strongly Agree (rated out of 5):

- 'I found the posters really useful': $4.5 / 5$

- 'I found the presentations very useful': $4.6 / 5$

- 'I will share what I've learnt about safety': 4.6/5 
Open space questions and word cloud responses also highlighted qualitative feedback with most frequent responses including 'inspiring', 'interesting and 'stimulating'.

174 posters were presented with good representation from all services and staff groups across the trust (18 on safer use of medicines, 15 on co-production, 52 on quality improvement, 50 on COVID and non-COVID safety, 16 on use of technology, 23 on supporting and involving staff). These posters have since been downloaded 4062 times.

Conclusion. The first CNWL safety conference proved an excellent opportunity to celebrate achievements in patient safety in a very difficult year. It was very well-received and well-attended by staff, promoting maximal learning across the organisation.

Whistle while you work: improving psychiatry training in a London NHS Trust and what we learned along the way

Emily Duncan*, Alex Bailey, Simon Edwards, Alison Butler and Layth Humsi

Central and Northwest London NHS Trust

${ }^{*}$ Corresponding author.

doi: 10.1192/bjo.2021.383

Aims. The aim of this project is to improve the training experience of Psychiatry trainees across CNWL. In QI terms, we want to achieve a satisfaction rating of above $7 / 10$ for all themes identified by August 2021.

Method. Collected baseline data on satisfaction and priority ratings on 7 training themes Held discussion groups with trainees for specific themes to generate issues and solutions Developed and provided Quality Improvement training for trainees and trainers, 1:1 support and QI clinics - empowering trainees to develop their own local project and to make changes to issues on the ground Enacted central changes in communication, responsiveness, recognising success.

Reassessed and fedback to the trainees throughout.

Result. Our baseline satisfaction survey was completed in June 2020. Trainees their satisfaction for each theme out of 10 and to rank their priorities for change. Results showed satisfaction was lowest in morale and in safety and highest in education and supervision. Their highest priorities for change were safety, then morale, with induction as the lowest priority.

We repeated the survey in October 2020. This showed improvements in most themes (apart from induction, perhaps due to induction having to be delivered virtually). Satisfaction in key priority areas of morale and safety increased from 4.53 to 6.37 , and 5.12 to 6.70 respectively. We also asked what 'one thing' would they improve about their training. Key phrases included teaching, on-call, communication and induction.

From this data, and softer feedback from trainees, it is encouraging that we are moving in a positive direction, but we are continuing to make changes.

Conclusion.

- Trainees must be central to the work in improving their training

- Using QI methodology helps - developing a structure and breaking down a bigger task helps make a plan

- Feedback is key - but people are busy and receive a lot of emails and requests to fill surveys - catching people 'in person' (virtually) was the best way to ensure a lot of responses

- Trainees have loads of great ideas, but they need support, time and resources to be able to develop their projects and changes
- Flexibility is crucial: some topics work better locally, driven by trainees and some require a more coordinated, central role

We hope that developing a structured approach to a large task like improving training will help make changes sustainable, and enables us to share our learning with others.

\section{Tackling constipation in patients on high dose antipsychotics}

Sandhya Eappen*

Parklands Hospital

${ }^{*}$ Corresponding author.

doi: 10.1192/bjo.2021.384

Aims. The aim of this audit was to look into patients on high dose antipsychotics who had developed complications of constipation in the PICU setting .

Background. Antipsychotics are usually used in the treatment of Schizophrenia and other psychotic illnesses. Drug such as Clozapine mainly has a higher risk profile due to gastrointestinal hypo motility. It could present as constipation, fecal impaction or a bowel obstruction and could even lead to death.

Method. During ward rounds enquired on bowel habits and diet.

Physical examination of patients complaining of abdominal pain.

Screened notes in past to see how many patients complained of constipation and interventions suggested and used.I65.

Result. 3 of the 10 patients on PICU were on high dose antipsychotics and 2 of them had reported constipation. Of which one required daily review and vigorous treatment with laxatives and dietary changes.

Recommendation

Bristol stool chart introduced as part of care plan for all patients.

Teaching presentation of constipation and its treatment management was given to the PICU team.

Involving medical team early on for assessment and prophylactic laxatives prescription.

Liaison with the pantry team to include more options of fruits and vegetables into daily meal plan for patients.

Data and material handed over to next trainee to Re-audit and complete audit cycle.

Conclusion. Appropriate prevention and early management of side effects can enhance the benefits of antipsychotics. Bowel function monitoring and the use of prophylactic laxatives for patient on high dose antipsychotics such as clozapine is advisable to prevent complications related to it.

\section{Bridging the gap - MTI experience to an excellent MTI experience - An experiential, theme driven, concordance verifying study}

Arun Enara ${ }^{1 \star}$, Kabir Garg ${ }^{2}$ and Ramachandran Kanchana ${ }^{1}$

${ }^{1}$ Hertfordshire partnership Foundation NHS trust and ${ }^{2}$ Oxleas NHS Foundation Trust

${ }^{*}$ Corresponding author.

doi: 10.1192/bjo.2021.385

Aims. To collate experiences of international medical graduates (trained psychiatrists) on the Medical Training Initiative (MTI) and equivalent programs (International Medical Fellowship 